\title{
Traduction et adaptation d'un modèle du jugement clinique infirmier pour la recherche et la formation infirmière en contexte francophone
}

\author{
Patrick Lavoie \\ Université de Montréal, patrick.lavoie.1@umontreal.ca \\ Marie-France Deschênes \\ Université de Montréal, marie-france.deschenes@umontreal.ca \\ Valérie Richard \\ Université de Montréal, valerie.richard.2@umontreal.ca \\ Jacinthe I. Pepin \\ Université de Montréal, jacinthe.pepin@umontreal.ca \\ Christine A. Tanner \\ Oregon Health \& Science University, tannerc@ohsu.edu \\ Kathie Lasater \\ Oregon Health \& Science University, lasaterk@ohsu.edu
}

Follow this and additional works at: https://qane-afi.casn.ca/journal

Part of the Adult and Continuing Education Commons, Cognition and Perception Commons, and the Nursing Commons

\section{Recommended Citation}

Lavoie, Patrick; Deschênes, Marie-France; Richard, Valérie; Pepin, Jacinthe I.; Tanner, Christine A.; and Lasater, Kathie (2021) "Traduction et adaptation d'un modèle du jugement clinique infirmier pour la recherche et la formation infirmière en contexte francophone," Quality Advancement in Nursing Education - Avancées en formation infirmière: Vol. 7: Iss. 2, Article 4.

DOI: https://doi.org/10.17483/2368-6669.1272

This Article is brought to you for free and open access by Quality Advancement in Nursing Education - Avancées en formation infirmière. It has been accepted for inclusion in Quality Advancement in Nursing Education - Avancées en formation infirmière by an authorized editor of Quality Advancement in Nursing Education - Avancées en formation infirmière. 


\section{Traduction et adaptation d'un modèle du jugement clinique infirmier pour la recherche et la formation infirmière en contexte francophone}

\section{Cover Page Footnote}

Nous tenons à remercier les expertes ayant commenté différentes versions du modèle traduit et adapté : Jacinthe Beauchamp (Université de Moncton, Canada), Anne Bourbonnais (Université de Montréal, Canada), Lisa Gonzalez (College of Southern Maryland, États-Unis), Kathy Holloway (Victoria University of Wellington, Nouvelle-Zélande), Mary Ann Jessee (Vanderbilt University, États-Unis), Michelle Kelly, (Curtin University, Australie), Janet Lusk Monagle (Endicott College, États-Unis) et Ann Nielsen (Oregon Health \& Science University, États-Unis). Nous adressons des remerciements spéciaux à Leticia Urrutia (Université de Montréal, Canada) pour les discussions qui ont permis d'imaginer ce projet. We would like to thank the experts who commented on different versions of the translated and adapted model: Jacinthe Beauchamp (Université de Moncton, Canada), Anne Bourbonnais (Université de Montréal, Canada), Lisa Gonzalez (College of Southern Maryland, USA), Kathy Holloway (Victoria University of Wellington, New Zealand), Mary Ann Jessee (Vanderbilt University, USA), Michelle Kelly, (Curtin University, Australia), Janet Lusk Monagle (Endicott College, USA), and Ann Nielsen (Oregon Health \& Science University, USA). Special thanks to Leticia Urrutia (Université de Montréal, Canada) for the discussions that helped us envision this project. 
Il y a près de 20 ans, Diekelman et Ironside (2002) soulevaient l'impératif d'appuyer l'innovation en formation infirmière sur des données issues de la recherche. Aujourd'hui, la formation infirmière est plus que jamais fondée sur un corpus de connaissances scientifiques sur ce que signifie apprendre à penser comme une infirmière ou un infirmier et la manière de faciliter cet apprentissage (Gresley, 2009). Pour poursuivre ces avancées, il importe de questionner les pratiques, de développer des connaissances fiables, et d'en évaluer la valeur et la pertinence afin de contribuer à l'évolution de la science et de la pratique de la formation infirmière (Oermann, 2007). Lorsqu'ils sont structurés et rigoureux, ces efforts peuvent mener à l'élaboration de guides de pratiques, de cadres de référence ou de théories qui orienteront la pratique et la recherche en formation infirmière. Or, la communauté francophone est souvent désavantagée par le fait que l'anglais reste la langue dominante dans le domaine des sciences (Ammon, 2011). La diffusion de connaissances pertinentes pour la formation infirmière en français demeure un enjeu important.

Les travaux rapportés dans cet article ont été entrepris pour favoriser la diffusion et la compréhension d'un cadre de référence largement utilisé en formation infirmière : le modèle du jugement clinique infirmier de Tanner (2006). Depuis sa publication en 2006, il a guidé un nombre important de travaux de recherche et continue d'orienter la formation infirmière au Canada et ailleurs dans le monde. Or, depuis sa publication en anglais, il n'a pas fait l'objet d'une traduction en français. Notre objectif était donc de traduire et d'adapter le modèle du jugement clinique infirmier de Tanner (2006) et d'en valider la version française.

\section{Contexte théorique}

Sommairement, le concept de jugement clinique peut être défini comme l'évaluation de différentes alternatives ou possibilités concernant la santé d'une personne (Thompson et al., 2013). Déterminer si l'état de santé d'une personne se détériore ou non est un exemple de jugement clinique, tout comme l'évaluation de la cause d'un ulcère des membres inférieurs. Le concept de raisonnement clinique réfère aux processus de pensée qui précèdent un jugement clinique. La distinction entre ces deux concepts sera approfondie plus loin dans cet article.

Historiquement, des courants théoriques issus de domaines scientifiques variés ont influencé la recherche sur ces deux concepts et, plus spécifiquement, l'intérêt pour les processus de raisonnement analytiques et intuitifs. Du point de vue analytique, les théories de l'analyse décisionnelle (Howard, 1966; Von Neumann et Morgenstern, 1947) abordent ces concepts sous un angle statistique, où chaque possibilité est décrite et sa probabilité d'occurrence est quantifiée. Le choix d'une alternative dépend de l'analyse de ces probabilités et de leur degré d'utilité prévue dans la résolution d'un problème. Ensuite, la théorie du traitement de l'information de Newell et Simon (1972) décrit un processus itératif de collecte de données et d'évaluation des possibilités d'actions par un raisonnement hypothético-déductif. Cette théorie se fonde sur des postulats cognitivistes concernant la capacité limitée de la mémoire à court terme à traiter simultanément des données; l'emmagasinement de groupements d'informations dans la mémoire à long termeparfois appelés patterns, schémas ou scripts_-permet de contrecarrer cette limite.

Le courant intuitif-humaniste, quant à lui, aurait été propulsé par les recherches de Benner (1984) sur le développement de l'expertise infirmière (Banning, 2008). En appliquant un modèle de développement de l'expertise (Dreyfus et Dreyfus, 1980) à la pratique infirmière, Benner (1984) décrit un passage d'une vision du monde se basant sur des connaissances théoriques à une compréhension holistique des situations fondée sur des connaissances acquises à travers l'expérience. Aujourd'hui, l'intuition est un élément central des théories du double processus du 
raisonnement (Evans, 2008; Kahneman, 2011), notamment la théorie du continuum cognitif (Hammond, 1996; Hammond et al., 1987). Ces théories suggèrent que les modes de raisonnement intuitifs et analytiques constituent deux pôles d'un même continuum. Les individus n'opéreraient pas selon un mode strictement intuitif ou analytique; leur raisonnement combinerait plutôt ces deux modes, à différents degrés, selon les caractéristiques des situations rencontrées.

Ces courants théoriques ont influencé le développement des connaissances sur le jugement et le raisonnement clinique infirmier. Nous avons repéré six modèles associés à ces concepts et émanant de travaux en sciences infirmières. D'abord, le modèle Outcome-Present state-Test (Pesut et Herman, 1998) décrit le raisonnement clinique comme une comparaison entre l'état actuel d'une personne et les résultats escomptés des soins. En plus d'allier des éléments des théories de l'analyse décisionnelle et du traitement de l'information, ce modèle soulève l'importance de la réflexion sur les stratégies cognitives. Il inclut aussi une composante narrative pour comprendre en profondeur une situation clinique et adopter une pensée critique dans l'exercice de la profession infirmière.

Ensuite, O’Neill et al. (2005) ont développé un modèle afin de diriger l'élaboration d'un système d'aide à la décision informatisé. Basé sur les théories de l'analyse décisionnelle et du traitement de l'information, il décrit la prise de décision infirmière et précise les caractéristiques du raisonnement des novices. Étroitement aligné avec les classifications et taxonomies de soins infirmiers, il arrime différentes composantes, notamment la collecte de données, l'évaluation des risques, l'influence du contexte, la génération d'hypothèses et les actions infirmières. Doté d'un caractère plus intégrateur que le modèle de Pesut et Herman (1998), il place un accent important sur l'utilisation d'hypothèses comme guide pour l'évaluation et l'intervention et n'aborde pas les processus intuitifs. Par ailleurs, les données relatives à son utilisation pour la formation infirmière sont rares.

Levett-Jones et al. (2010) ont élaboré un modèle du raisonnement clinique en huit étapes : considérer un patient ou une patiente, rassembler des informations, les traiter, identifier un problème, établir des buts, intervenir, évaluer les résultats et réfléchir sur le processus afin d'apprendre. Pour l'opérationnaliser, ils proposent cinq «bons » du raisonnement clinique, soit la capacité de reconnaître les bons signaux et de prendre les bonnes décisions pour le bon patient ou la bonne patiente, au bon moment et pour la bonne raison. Ce modèle accorde beaucoup d'importance aux stratégies cognitives et remet à l'individu l'évaluation de ce qui est considéré comme «bon ». Or, il est souvent rapporté que les novices arrivent difficilement à identifier les éléments saillants d'une situation (O’Neill et al., 2005; Simmons, 2010; Tanner, 2006). Ainsi, il deviendrait probablement difficile pour eux de reconnaître les «bons signaux » et d'évaluer convenablement les choix qui s'offrent à eux.

Plus récemment, Lee et al. (2016) ont défini un modèle cyclique du raisonnement clinique en cinq étapes rappelant la démarche de soins infirmiers : collecte de données, analyse, diagnostic, planification et évaluation. Pour chaque étape, ils précisent les stratégies cognitives qui sont utilisées par les infirmières et les infirmiers, la validation des données et la détection de patterns étant respectivement la plus et la moins fréquemment utilisées. Pour leur part, Huang et al. (2018) ont suggéré quatre domaines de compétences associés au raisonnement clinique infirmier: la conscience des signaux cliniques, la confirmation des problèmes cliniques, la détermination et l'implantation des actions, et l'évaluation et réflexion. Il est à noter que ces deux modèles sont basés sur des études qui visaient à opérationnaliser le raisonnement clinique, que ce soit par des stratégies cognitives ou des indicateurs de compétences. 
Auparavant, Tanner (2006) a conçu un modèle du jugement clinique infirmier. Ce modèle se fonde sur cinq conclusions tirées par l'autrice à partir de diverses études qu'elle a recensées. Prenant appui sur ces conclusions, Tanner (2006) a développé un modèle en quatre phases dynamiques qui, contrairement aux autres modèles, reconnaît que les jugements cliniques sont influencés par le contexte, les valeurs et les biais de l'infirmière ou de l'infirmier et sa relation avec la personne. Le modèle explique que ces éléments jouent sur ce que l'infirmière ou l'infirmier remarque, les données additionnelles recueillies, son choix d'impliquer la personne dans sa prise de décision et les options envisagées. Il décrit une nécessité constante de réévaluer et de modifier ses réponses dans les situations complexes qui évoluent rapidement.

Le modèle de Tanner (2006) est particulièrement intéressant en raison de son caractère intégrateur et du fait qu'il résume plus de 20 ans de recherche. Il ne se rattache pas spécifiquement à une des théories décrites précédemment, mais propose plutôt d'en combiner des éléments; par conséquent, il englobe les processus de raisonnement analytiques et intuitifs. Aussi, le modèle affirme la place de l'expérience comme source de développement du jugement clinique infirmier par la réflexion. Ce dernier concept est une avenue de formation particulièrement intéressante qui a pris un essor important dans la formation infirmière (Dubé et Ducharme, 2015; Goulet et al., 2016). Tanner (2006) suggère que son modèle soit utilisé pour décrire le jugement clinique d'infirmières et d'infirmiers de différents niveaux d'expertise et pour guider la création d'expériences d'apprentissage dans la formation en soins infirmiers.

\section{Méthode}

Notre démarche s'est inspirée de recommandations pour la traduction, l'adaptation et la validation transculturelle d'instruments de mesure (Sousa et Rojjanasrirat, 2011). Ces recommandations s'appuient sur une synthèse des approches méthodologiques les plus complètes, rigoureuses et éprouvées pour produire une traduction qui préserve la dénotation et la connotation de la version originale d'un instrument de mesure. Dans notre cas, l'objet de la traduction était un modèle qui, contrairement à un instrument de mesure, ne comporte pas d'items ou de propriétés psychométriques. Ainsi, nous avons appliqué les quatre premières étapes recommandées par Sousa et Rojjanasrirat (2011), qui sont illustrées à la Figure 1. Cette démarche a été autorisée par l'autrice originale du modèle du jugement clinique infirmier, qui a participé à la rédaction du présent article. 


\section{Figure 1}

Démarche de traduction du modèle

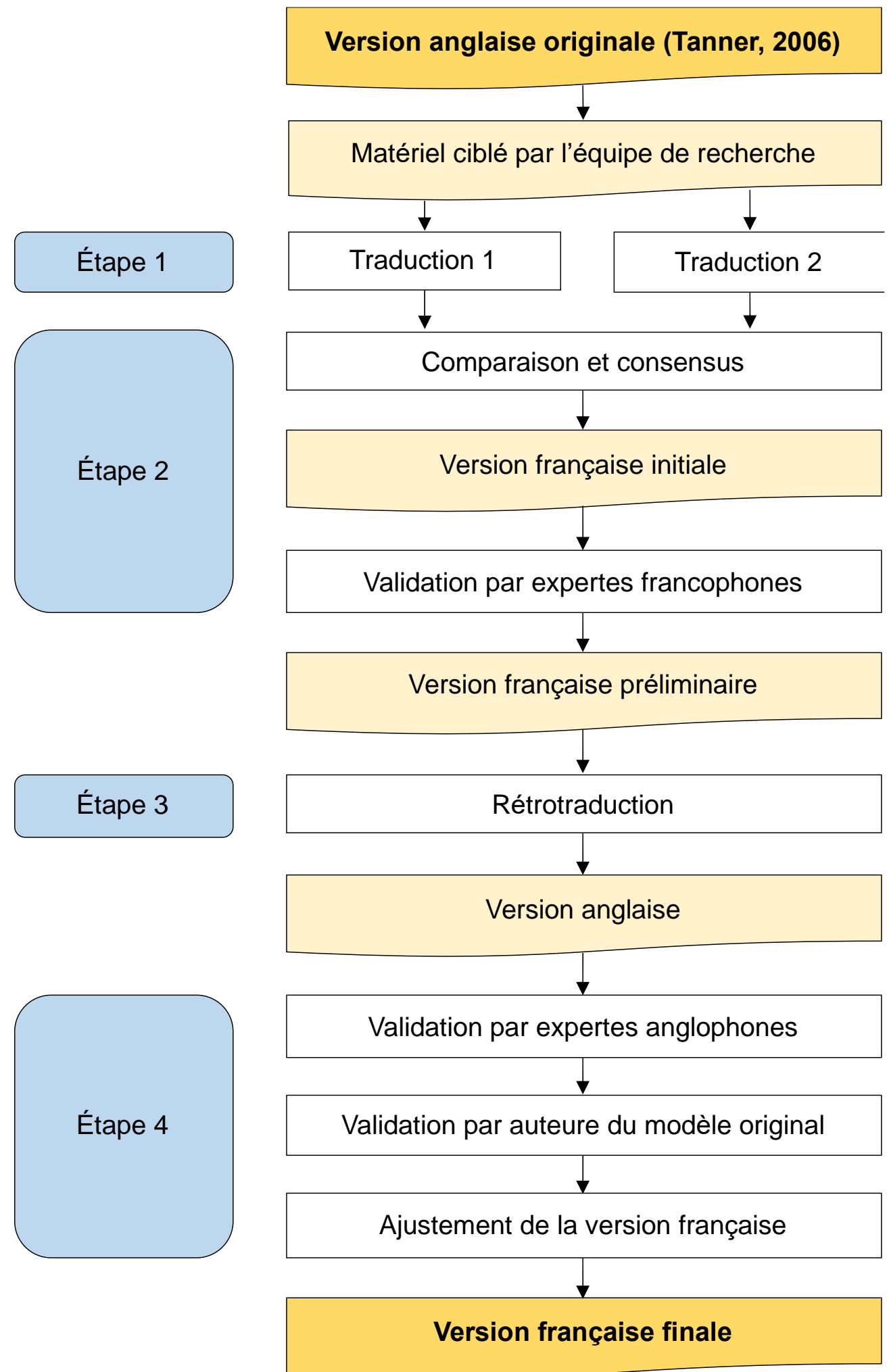

Note. Adaptée de Sousa et Rojjanasrirat (2011) 


\section{Préparation du matériel}

Pour débuter, nous avons examiné l'article original de Tanner (2006) et ciblé les sections à traduire. Outre la section décrivant le modèle (p. 208-209), nous avons conclu qu'il était nécessaire de traduire les définitions des termes « jugement clinique » et « raisonnement clinique » (p. 204205), de même que les cinq conclusions (p. 204-205).

\section{Première étape : double traduction de la langue source vers la langue cible}

Trois membres de l'équipe de recherche (PL, MFD, VR) ont traduit le matériel ciblé de l'anglais (langue source) vers le français (langue cible). Ces trois personnes étaient d'origine québécoise et avaient pour langue maternelle le français. PL et MFD étaient familiers avec la terminologie en formation infirmière, ainsi qu'avec le modèle. VR était familière avec le domaine des sciences infirmières, mais pas avec la terminologie en formation infirmière ni avec le modèle.

À cette étape, deux versions françaises du matériel ont été produites de manière indépendante : PL et VR ont travaillé en équipe et MFD a travaillé individuellement. Ils ont tenté de préserver les idées du modèle et de respecter la formulation originale des sections ciblées. Toutefois, l'article de Tanner (2006) était présenté sous forme de recension des écrits et faisait référence à des textes publiés il y a 15 à 40 ans au moment de la traduction. Ainsi, les membres de l'équipe de recherche ont reformulé les phrases qui référaient à d'autres écrits de manière à conserver uniquement les notions à la base du modèle. Cette manœuvre avait aussi pour but de réduire la longueur du texte et de mettre en exergue les segments jugés les plus importants pour saisir le sens du modèle.

\section{Étape 2 : comparaison des deux traductions}

Par la suite, les deux versions françaises ont été comparées par les trois membres de l'équipe de recherche impliqués dans leur traduction afin de résoudre toutes ambiguïtés et divergences dans les mots et les phrases employés. À la suite de l'atteinte d'un consensus, il et elles ont construit une version française initiale du modèle. Pour ce faire, il et elles ont regroupé les idées similaires et organisé les sections afin d'assurer la fluidité du texte. Ainsi, la structure et l'organisation de cette version différaient de l'article original de Tanner (2006).

Cette version française initiale a été présentée à trois expertes en sciences infirmières francophones (JP, JB, AB) œuvrant en milieux universitaires canadiens et familières avec le modèle original. Ces expertes avaient pour tâche de lire et de commenter individuellement la version française initiale pour déterminer si le vocabulaire employé était juste et si les idées de l'article original étaient correctement véhiculées. À partir de leurs commentaires, la version française initiale a été raffinée en vue d'élaborer une version française préliminaire du modèle.

\section{Étape 3 : rétrotraduction}

La version française préliminaire a fait l'objet d'une rétrotraduction par une firme de traduction indépendante, spécialisée dans le domaine de la santé. Un traducteur bilingue dont la langue maternelle était l'anglais et qui n'était pas familier avec le modèle original a été impliqué à cette étape, ce qui a résulté en la production d'une version anglaise du modèle.

\section{Étape 4 : comparaison avec la version originale}

La version anglaise a été transmise à sept professeures en sciences infirmières de l'Australie (MK), des États-Unis (LG, MAJ, KL, JLM, AN) et de la Nouvelle-Zélande (KH). Ces expertes ont été sollicitées en raison de leur connaissance du modèle de Tanner (2006) qu'elles avaient étudié 
dans le cadre de leur formation à la recherche. Elles étaient donc très familières avec ses composantes, en plus de l'avoir utilisé à plusieurs reprises dans leurs activités d'enseignement et de recherche. Elles ont été invitées à relire l'article original, puis à commenter individuellement la version anglaise issue de la rétrotraduction. Elles ont vérifié l'équivalence au niveau conceptuel, au niveau sémantique et du contenu entre ces deux versions. Deux membres de l'équipe de recherche (PL, KL) ont compilé leurs commentaires et effectué les ajustements suggérés à la version anglaise.

Cette version anglaise ajustée a été présentée à l'autrice originale (CAT). Elle a été invitée à commenter la structure et le vocabulaire employé, ainsi qu'à s'assurer que la présentation des idées et des concepts reflétait bien sa vision. Ses commentaires ont servi à raffiner la version anglaise ajustée. Par la suite, la version française préliminaire a été revisitée et ajustée dans son ensemble afin de produire la version française finale, présentée dans son intégralité dans la section suivante.

\section{Un modèle du jugement clinique infirmier basé sur la recherche}

Le modèle proposé par Tanner (2006) s'appuie sur une synthèse de près de 200 écrits scientifiques sur le jugement clinique et d'autres concepts apparentés. À partir de ces écrits, Tanner (2006) a tiré cinq conclusions qui seront détaillées plus bas : 1) Le bagage des infirmières et infirmiers influence davantage leurs jugements cliniques que les données objectives d'une situation de soins; 2) Un jugement clinique solide repose, dans une certaine mesure, sur une connaissance de la personne et de sa façon habituelle de réagir, ainsi que sur l'engagement d'une infirmière ou d'un infirmier à l'égard de la personne et ses préoccupations; 3) Les jugements cliniques sont influencés par le contexte, notamment par la culture du milieu de soins et le contexte politique et social; 4) Les infirmières et infirmiers mettent en œuvre un mode de raisonnement ou en combinent plusieurs; 5) La réflexion sur l'action fait généralement suite à un événement déclencheur et est essentielle pour le développement des connaissances et l'amélioration du jugement clinique et du raisonnement clinique.

En s'appuyant sur ces conclusions, Tanner (2006) a construit un modèle qui fait office de cadre de référence pour la formation et la recherche en sciences infirmières (Figure 2). Bien que le modèle décrive le jugement clinique d'infirmières et infirmiers d'expérience, il offre un vocabulaire pour décrire le développement des étudiantes et étudiants en sciences infirmières. De cette façon, il permet de guider l'accompagnement dans l'identification et l'articulation des éléments qu'ils souhaitent maintenir ou améliorer, de même que le développement de situations d'apprentissage susceptibles de les aider en ce sens. 


\section{Figure 2}

\section{Modèle du jugement clinique infirmier de Tanner (2006)}

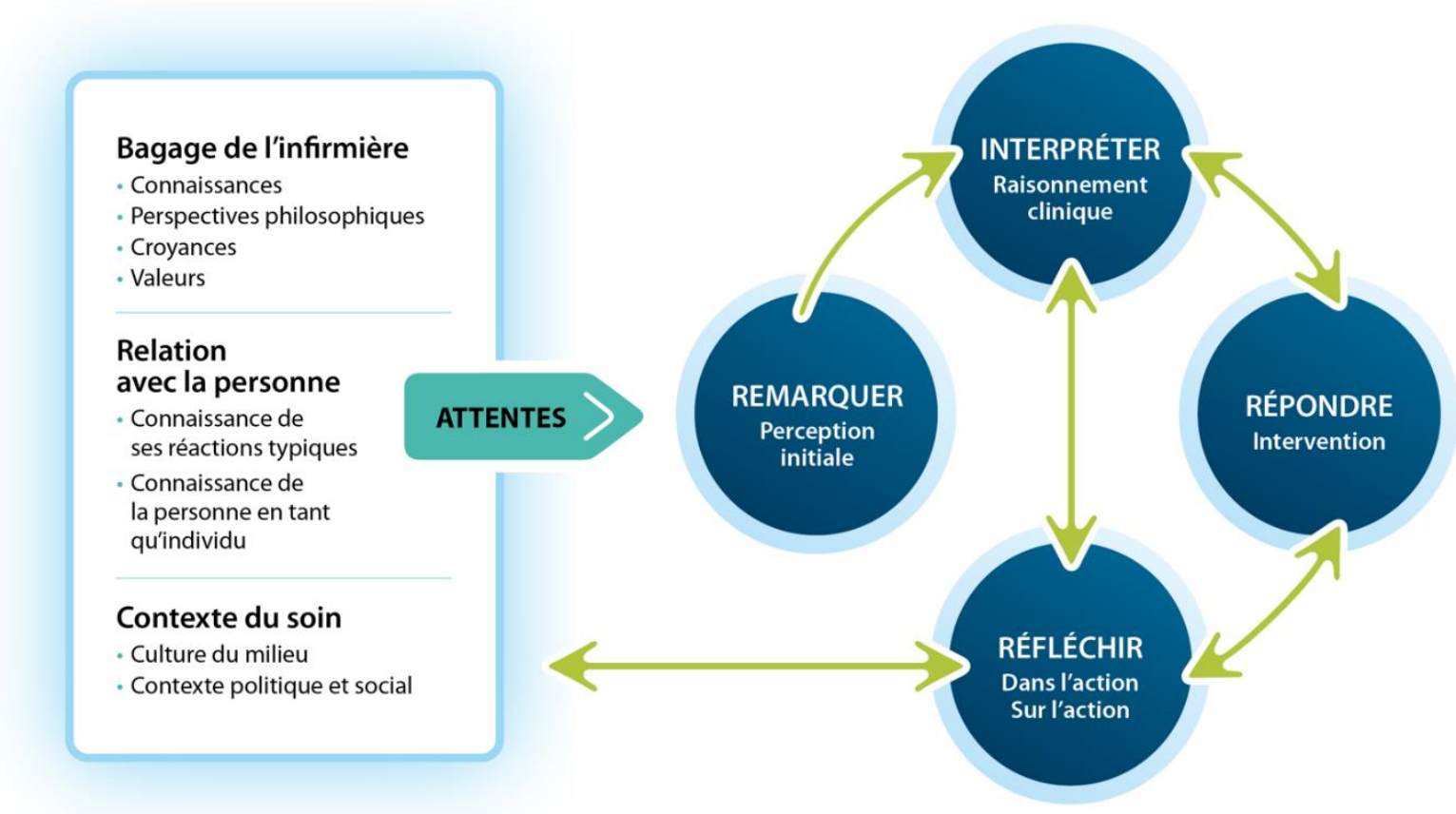

Note. Traduit et modifié avec l'accord de l'autrice.

En sciences infirmières, les termes « jugement clinique », « résolution de problèmes », «prise de décision» et «pensée critique » sont souvent utilisés de façon interchangeable. Néanmoins, dans le modèle de Tanner (2006), le terme « jugement clinique » est défini comme une compréhension, un constat ou une conclusion relative aux besoins, aux préoccupations ou aux problèmes de santé d'une personne. Ce terme peut aussi désigner une décision d'agir, c'est-à-dire le choix d'appliquer ou d'adapter une approche reconnue, d'en initier une nouvelle, ou encore de ne rien faire, tout dépendant de la réaction de la personne. Par ailleurs, il importe de distinguer le jugement clinique de la démarche de soins infirmiers. Cette démarche est un modèle de résolution de problème qui peut être utile pour les étudiantes et étudiants qui en sont à leurs débuts. Toutefois, elle comprend des étapes qui sont présentées de manière linéaire et systématique, ce qui ne reflète pas la complexité du raisonnement clinique des infirmières et infirmiers — débutants ou experts — ni les multiples facteurs interreliés qui l'influencent.

Le « raisonnement clinique » désigne l'ensemble des processus cognitifs qui précèdent un jugement clinique. Ce raisonnement peut prendre une forme analytique, lorsque les infirmières et infirmiers formulent des hypothèses et les évaluent au regard des données d'une situation clinique et de résultats probants. Il peut aussi prendre une forme engagée ou pratique, lorsque les infirmières et infirmiers reconnaissent intuitivement un portrait clinique ou un schéma typique. En raison de leur familiarité avec des situations similaires et de leur capacité de raisonner rapidement, ils perçoivent alors les caractéristiques saillantes de la situation, la comprennent à partir de leurs expériences antérieures et y répondent de façon automatique, sans avoir à y réfléchir très 
longuement. Écouter le récit qu'une personne fait de son expérience de santé peut aussi engager les infirmières et infirmiers dans un raisonnement de forme narrative, par lequel ils tentent de dégager ce que cette expérience signifie pour la personne.

Pour décrire le jugement clinique dans sa complexité, le modèle de Tanner (2006) comprend quatre aspects : remarquer, interpréter, répondre et réfléchir. Ces aspects s'appliquent dans toutes les situations, particulièrement celles qui peuvent évoluer rapidement et dont les paramètres sont ambigus ou mal définis. Dans ces moments dynamiques, les infirmières et infirmiers doivent continuellement évaluer la situation dans sa globalité, comprendre les changements observés et ajuster leurs réponses. Initialement, Tanner (2006) a présenté ces quatre aspects séparément des cinq conclusions qui sous-tendent son modèle. Néanmoins, nous avons choisi de les intégrer dans la présentation qui suit. Pour le lectorat, nous croyons que ceci illustre la manière dont les cinq conclusions s'arriment aux concepts du modèle. Pour une description exhaustive de ces conclusions, le lectorat est invité à consulter l'article original de Tanner (2006).

\section{Remarquer}

Remarquer, c'est prendre conscience d'un élément ou d'une caractéristique de la situation clinique qui mérite qu'on y porte attention, ce qui aide les infirmières et infirmiers à se construire une perception initiale de ce qui arrive à la personne. Cela ne fait pas nécessairement référence à la collecte de données - la première étape de la démarche de soins. En effet, cela dépend plutôt de la manière dont la situation clinique correspond ou ne correspond pas à leurs attentes, qui peuvent être définies comme leur compréhension, basée sur différents types de connaissances, de la manière dont la situation devrait se dérouler. Pour remarquer, une infirmière ou un infirmier doit percevoir un écart entre la situation clinique et ses attentes, que celles-ci soient explicites ou non. Le fait de remarquer est parfois associé à une impression de saillance (sense of salience), ce qui signifie que l'information pertinente ressort ou se démarque aux yeux des infirmières et infirmiers expérimentés, sans nécessairement qu'ils aient recours à des connaissances spécifiques.

Les attentes des infirmières et infirmiers et leur perception initiale découlent de : 1) leurs connaissances théoriques; 2) leurs connaissances expérientielles issues de la pratique ou de situations similaires; et 3) leur connaissance de la personne et de ses réactions habituelles ou typiques. Par exemple, les infirmières et infirmiers d'expérience en soins chirurgicaux connaissent les caractéristiques typiques de la douleur postopératoire (connaissances expérientielles). Ils comprennent aussi les mécanismes physiologiques et physiopathologiques de la douleur selon le type de chirurgie (connaissances théoriques). Lorsqu'ils prennent soin d'une personne en particulier, ils apprennent à connaître les caractéristiques uniques de sa douleur et ses réactions typiques face à celle-ci (connaissance de la personne). Ces trois types de connaissances façonnent leurs attentes par rapport aux caractéristiques ou à l'intensité de la douleur de cette personne. Si la situation ne correspond pas à leurs attentes, ils le remarqueront et se formeront une perception initiale du problème immédiat.

Outre les attentes des infirmières et infirmiers, d'autres facteurs influencent ce qu'ils sont susceptibles de remarquer dans une situation clinique, notamment leur bagage, incluant leurs valeurs et leur vision de l'excellence dans les soins, leur relation avec la personne et le contexte de l'environnement de soins. Les éléments liés à chacun de ces facteurs sont développés ci-après. 


\section{Bagage de l'infirmière ou l'infirmier}

Conclusion $n^{0} 1$ : Le bagage des infirmières et infirmiers - leurs connaissances, leur perspective philosophique, leurs croyances et leurs valeurs - influence davantage leurs jugements cliniques que les données objectives d'une situation de soins.

Les jugements cliniques sont empreints de la perspective philosophique ou des valeurs des infirmières et infirmiers, c'est-à-dire ce qu'ils considèrent bien et juste. Ces croyances orientent, souvent inconsciemment, la façon dont ils abordent une situation : ce qu'ils remarquent, les actions ou les interventions qu'ils considèrent et, ultimement, les décisions qu'ils prennent. À titre d'exemple, Tanner (2006) décrit les résultats d'une étude de McCarthy (2003) qui révèlent que la perspective des infirmières et infirmiers sur le vieillissement influence leur capacité à détecter un syndrome confusionnel aigu chez la personne âgée. Dans le même ordre d'idée, leur attitude à l'égard de la douleur et la valeur qu'ils attribuent au confort influencent la manière dont ils gèrent la douleur. Ainsi, la gestion infirmière du syndrome confusionnel aigu ou de la douleur peut être entendue comme une question morale; les valeurs et les attitudes des infirmières et des infirmiers, de même que les obstacles politiques ou institutionnels qu'ils rencontrent, influencent leurs actions - parfois davantage que leur compréhension de l'expérience de la personne.

Par ailleurs, le jugement clinique s'appuie sur différents types de connaissances, soit des connaissances théoriques, des connaissances expérientielles et des connaissances de la personne - qui sont étroitement liées aux modes de raisonnement décrits dans les prochaines sections. Dérivées de la science et de la théorie, les connaissances théoriques sont abstraites, généralisables et applicables dans de nombreuses situations. Souvent qualifiées de tacites, les connaissances expérientielles se construisent à travers la pratique et l'expérience des infirmières et infirmiers. Ces savoirs facilitent la reconnaissance de portraits cliniques ou de schémas typiques. Enfin, les connaissances de la personne relèvent d'une compréhension approfondie et partagée de son expérience et de ses attentes par rapport aux soins. Ces trois types de connaissance opèrent souvent de manière simultanée et ne peuvent pas toujours être distingués.

Pour les infirmières et infirmiers d'expérience qui rencontrent une situation familière, les connaissances nécessaires sont facilement activées et mobilisées. Ils sont en mesure de répondre rapidement et de façon intuitive à la situation. Devant la même situation, les infirmières et infirmiers débutants raisonnent souvent de façon analytique. Ils doivent déterminer, parmi leurs connaissances théoriques et leurs expériences de vie, celles qui seront utiles pour comprendre la situation. Ceci les amène à développer des connaissances expérientielles qui leur permettront d'affiner, d'enrichir et d'adapter leurs connaissances théoriques aux particularités des situations qu'ils rencontrent.

\section{Relation avec la personne}

Conclusion $n^{0} 2$ : Un jugement clinique solide repose, dans une certaine mesure, sur une connaissance de la personne et de sa façon habituelle de réagir, ainsi que sur l'engagement d'une infirmière ou d'un infirmier à l'égard de la personne et ses préoccupations

La «connaissance de la personne » est au cœur du jugement clinique des infirmières et infirmiers. Souvent tacite, ce type de connaissance est difficile à mettre en mots; néanmoins, les infirmières et infirmiers y réfèrent fréquemment. Cette compréhension se construit dans la relation avec la personne, lorsqu'ils l'observent, écoutent le récit de son expérience de santé et en viennent à comprendre ses réactions typiques. 
Au-delà de l'évaluation clinique, cette compréhension profonde de la personne influence le jugement clinique et réfère à deux manières de la connaître. Premièrement, cela implique de connaître la façon dont la personne réagit habituellement à certaines situations ou à certains soins, c'est-à-dire ses réactions typiques ou attendues. Par exemple, une infirmière ou un infirmier pourrait savoir qu'une personne devient somnolente lorsqu'elle est en hypoglycémie, alors qu'une autre présente une sudation abondante. En connaissant les réactions typiques d'une personne, certains aspects de sa situation ressortent et deviennent plus saillants, tandis que d'autres paraissent moins importants. Il est possible de distinguer ces nuances en comparant le portrait actuel de la personne à son portrait habituel ou typique. Deuxièmement, connaître la personne en tant qu'individu implique de connaître les caractéristiques qui la rendent unique, comme ses préférences et ses valeurs. Ceci permet d'individualiser la réponse de l'infirmière ou de l'infirmier et ses interventions.

\section{Contexte du soin}

Conclusion no 3 : Les jugements cliniques sont influencés par le contexte, notamment par la culture du milieu de soins et le contexte politique et social.

Les infirmières et infirmiers ont tendance à se mettre au diapason de la culture de leur milieu de soins et de leur groupe de travail ; ils développent une compréhension partagée des normes, des habitudes et des routines de travail. Dans une certaine mesure, ces éléments déterminent les situations dans lesquelles on s'attendra à ce qu'ils posent un jugement clinique. De plus, ces éléments influencent les connaissances qui sont valorisées par les membres de ce groupe et les habiletés qu'ils sont susceptibles de se transmettre.

Le contexte politique et social influence aussi le jugement clinique. Par exemple, les relations interprofessionnelles ou interdisciplinaires — notamment les inégalités de statut et les différences de pouvoir entre les infirmières ou infirmiers et les médecins — influencent le degré auquel ils se sentent habilités à comprendre et à agir devant un problème.

\section{Interpréter}

Après avoir remarqué un élément ou une caractéristique de la situation, les infirmières et infirmiers s'engagent dans un processus d'évaluation ciblé qui découle de leur perception initiale. Ils évaluent la situation et revisitent leur perception initiale jusqu'à ce qu'ils développent une compréhension suffisante de la situation pour être en mesure d'y répondre.

Conclusion $n^{0} 4$ : Les infirmières et infirmiers mettent en œuvre un mode de raisonnement ou en combinent plusieurs.

Le raisonnement clinique des infirmières et infirmiers d'expérience peut suivre au moins trois grandes modes : 1) les processus analytiques, 2) l'intuition et 3) la pensée narrative. Dans chacun, il existe plusieurs modes distincts qui peuvent opérer seuls ou en combinaison. Il ressort des études réalisées à ce jour qu'aucun mode de raisonnement unique ne fonctionne dans toutes les situations et pour toutes les infirmières et tous les infirmiers de tous les niveaux d'expertise. En fait, ils combinent souvent différents modes de raisonnement pour interpréter une situation clinique. Le ou les modes de raisonnement sollicités dépendent des paramètres de la situation clinique, de la perception initiale (ce que l'infirmière ou infirmier a remarqué), du contexte du soin, du bagage de l'infirmière ou infirmier et de sa relation avec la personne.

Processus analytiques. Les processus analytiques consistent à décomposer une situation en ses éléments. Ils sont caractérisés par la génération et l'évaluation systématique et rationnelle 
d'hypothèses par rapport aux données cliniques ou aux résultats escomptés. L'infirmière ou l'infirmier tente de cibler l'hypothèse (ou les hypothèses) qui explique le mieux les données recueillies et qui suggère une réponse appropriée. Les processus analytiques sont généralement sollicités dans les circonstances suivantes : (a) un manque de connaissances de l'infirmière ou de l'infirmier; (b) un décalage ou un écart entre ses attentes et ce qui se passe dans la situation; (c) plusieurs hypothèses sont plausibles et il faut prendre une décision éclairée (un processus analytique permettra de peser les arguments en faveur de chaque hypothèse, ou les avantages et les inconvénients de chaque intervention).

Intuition. L'intuition se caractérise par une compréhension immédiate et automatique d'une situation clinique et découle des connaissances expérientielles issues de la pratique dans des situations similaires. Dans plusieurs études dont celle de Benner et al. (2009), cette compréhension d'une situation est nommée «recognition of a pattern», ce qui peut être traduit par la reconnaissance d'un « portrait clinique », d'un « schéma typique » ou d'un « profil attendu ».

Pensée narrative. La pensée narrative permet aux infirmières et infirmiers de comprendre une situation particulière et de trouver un sens à l'expérience de la personne à partir de ses préoccupations, de ses intentions et de ses motivations. C'est en interprétant les récits que les personnes font de leurs expériences de santé qu'il est possible de comprendre la signification qu'elles y attribuent, leurs manières de s'y adapter et la façon dont elles envisagent le futur. La pensée narrative amène une compréhension profonde de la personne en tant qu'individu, ce qui influence les actions et décisions infirmières. De surcroît, la narration est un outil de réflexion important : écouter ou raconter des histoires issues de son expérience en tant qu'infirmière ou infirmier aide à comprendre et à apprendre de son expérience.

\section{Répondre}

Répondre, c'est décider d'un plan d'action approprié pour la situation (qui peut consister à ne pas agir pour l'instant). La réponse - ou l'intervention — de l'infirmière ou infirmier découle directement de son interprétation de la situation.

Il est à noter que l'évaluation de la situation de la personne et la réponse de l'infirmière ou infirmier ont une double fonction : les deux perspectives soutiennent le raisonnement clinique et en sont le résultat. Par exemple, lorsqu'une infirmière ou un infirmier emploie un processus analytique, son évaluation de la situation de la personne l'amène à formuler des hypothèses, lesquelles sont constamment réévaluées pour décider de la réponse et des interventions les plus appropriées. Ce mode de raisonnement l'amène à approfondir son évaluation et sa compréhension de la situation clinique. Dans le même ordre d'idée, l'infirmière ou l'infirmier qui emploie un mode de raisonnement intuitif détermine sa réponse à partir des similitudes entre la situation actuelle et des situations vécues antérieurement. Pour vérifier si son intuition s'avère fondée, elle ou il évalue les réactions de la personne à ses interventions.

\section{Réfléchir}

Conclusion $\mathbf{n}^{\mathbf{0}} 5$ : La réflexion sur l'action fait généralement suite à un événement déclencheur, souvent une défaillance ou un écart (réel ou perçu) dans la pratique, et est essentielle pour le développement des connaissances et l'amélioration du jugement clinique et du raisonnement clinique.

Dewey (1933) définit la réflexion comme l'examen prolongé, délibéré et minutieux d'une croyance ou d'une connaissance à la lumière de ce qui la soutient et de ses conséquences. La 
réflexion implique un retour sur l'expérience, l'analyse des événements et la mise en relation des différentes données, du contexte, du bagage de l'infirmière ou infirmier et de sa relation avec la personne. La réflexion est essentielle pour le développement ou le raffinement des connaissances et l'amélioration du jugement clinique et du raisonnement clinique dans des situations complexes. De plus, la réflexion améliore l'apprentissage à partir de l'expérience. Chaque situation clinique est une opportunité d'apprentissage, à condition que les infirmières ou infirmiers soient dans un contexte favorable à la réflexion, qu'ils en aient l'opportunité et qu'ils aient développé les compétences et l'habitude de réfléchir sur leur pratique.

Le modèle du jugement clinique distingue deux formes de réflexion : la réflexion dans l'action et la réflexion sur l'action. Réfléchir dans l'action, c'est surveiller les réactions de la personne pendant les soins et les résultats des interventions. La réflexion dans l'action fait référence à la capacité de « lire » la personne — comment elle réagit aux actions des infirmières ou infirmiers - et à ajuster les soins en conséquence. Une grande partie de cette réflexion dans l'action est implicite, à moins que les résultats de soins attendus ne soient pas atteints.

Réfléchir sur l'action, c'est évaluer les résultats des actions en se concentrant sur la pertinence de tous les aspects précédents (ce qui a été remarqué, comment cela a été interprété et comment l'infirmière ou infirmier a répondu). En comparaison avec la réflexion dans l'action, la réflexion sur l'action est plus délibérée et a lieu après la situation. S'engager dans la réflexion sur l'action requiert un sens des responsabilités, puisque l'infirmière ou l'infirmier doit se sentir tenu de faire des liens entre ses actions et leurs résultats, de même qu'avec ses connaissances, sa perspective philosophique, ses croyances et ses valeurs. La réflexion requiert également une connaissance des résultats possibles, par laquelle l'infirmière ou l'infirmier parvient à distinguer ce qui résulte de ses actions.

\section{Discussion}

Cette démarche de traduction, d'adaptation et de validation, réalisée selon les meilleures pratiques en la matière, a permis de présenter une version française du modèle du jugement clinique infirmier de Tanner (2006). Cet exercice visait à promouvoir la science et la pratique de la formation infirmière, particulièrement dans les contextes où le développement des connaissances et le choix de stratégies pédagogiques dans les programmes francophones s'appuient sur des théories et modèles spécifiques de compétences infirmières, dont le jugement clinique.

Pour les formateurs, ce modèle s'inscrit en appui à différentes stratégies pédagogiques pour accompagner les infirmières et infirmiers dans les processus qui les mènent à poser un ou des jugements cliniques. Il propose un vocabulaire pour décrire ces processus et les différents éléments qui peuvent les influencer, ce qui contribue à les distinguer. Ce faisant, il pose un éclairage renouvelé sur le raisonnement et le jugement clinique infirmier et contribue à l'articulation de stratégies pédagogiques cohérentes avec ceux-ci. À titre d'exemple, le modèle a servi à l'élaboration de stratégies pour accompagner la réflexion des infirmières et des infirmiers, que ce soit en milieu clinique ou académique (Goudreau et al., 2015; Lavoie et al., 2017; Nielsen et al., 2007), et pour structurer diverses stratégies pédagogiques comme la simulation clinique, la cartographie conceptuelle et les études de cas (Huang et al., 2012; Lasater, 2007b). Bien que ces stratégies aient toutes le potentiel de contribuer au développement du jugement clinique infirmier, les résultats d'une revue systématique des écrits parus après la publication du modèle original n'ont pas permis de montrer la supériorité d'une d'entre elles (Cappelletti et al., 2014). Les autrices de cette revue systématique recommandent plutôt de les utiliser en combinaison pour maximiser leurs 
effets. Ayant observé que les études parues après le modèle de Tanner (2006) se concentraient sur le rôle de la formation dans le développement du jugement clinique infirmier, ces autrices recommandent de considérer une sixième conclusion pour bonifier le modèle : les stratégies pédagogiques visant l'amélioration du jugement clinique pourraient influencer le bagage de l'infirmière ou de l'infirmier (Cappelletti et al., 2014). Rappelons que, selon la première conclusion qui sous-tend le modèle, ce bagage influencerait davantage les jugements cliniques que les données objectives d'une situation de soins. Cette conclusion s'est d'ailleurs reflétée dans les résultats d'une étude récente, qui a montré que différentes composantes du bagage d'étudiantes infirmières et étudiants infirmiers influençaient ce qu'ils remarquaient dans une situation de soins, notamment leurs expériences antérieures, leur âge et leur genre (Lasater et al., 2019).

Pour les chercheurs, le modèle et sa version française offrent des avenues intéressantes pour sonder plus en profondeur l'évaluation de cette compétence. Notons qu'il a donné lieu à la conception d'une rubrique d'évaluation validée qui permet de quantifier différentes facettes des quatre aspects du modèle : remarquer, interpréter, répondre et réfléchir (Lasater, 2007a). Cette rubrique a d'ailleurs récemment été traduite et sa version française a été validée (Beauchamp et al., 2021). Par ailleurs, les travaux de Goudreau et al. (2014) et Boyer et al. (2015) se sont inscrits dans une conception développementale et ont permis de préciser des étapes de développement du raisonnement et du jugement clinique, permettant de mieux évaluer son développement (Boyer et al., 2015) et de repenser les mesures de soutien à son apprentissage dans les programmes de formation (Goudreau et al., 2014). Ce caractère développemental de la compétence suggère également d'autres avenues de recherche qui allient à la fois des modèles théoriques et des stratégies pédagogiques pour en favoriser le développement. Les stratégies pédagogiques basées sur la concordance de scripts sont un exemple de cet arrimage cohérent en formation infirmière (Deschênes et Goudreau, 2017). Sur le plan théorique, les scripts sont définis comme des réseaux de connaissances structurés dans la mémoire à long terme des cliniciens. La concordance de scripts consiste à comparer les jugements d'une étudiante ou d'un étudiant à ceux d'un panel de cliniciennes et cliniciens experts dans des situations apparentées à la pratique professionnelle, où les données sont complexes ou incomplètes. Les travaux sur cette stratégie s'inscrivent donc dans une perspective systémique et transformationnelle du développement du raisonnement et du jugement clinique, où les différents réseaux de savoirs de l'infirmière et infirmier sont construits, affinés et sollicités tout au long de sa formation, puis de sa carrière professionnelle. Cela n'est pas sans rappeler que divers modes de savoirs, interconnectés, permettent de soutenir le développement des connaissances de la discipline infirmière (Pepin et al., 2017). Les trois modes de raisonnement clinique décrits par Tanner (2006) - processus analytiques, intuition, pensée narrative - permettent de se représenter les manières par lesquelles les infirmières et infirmiers appréhendent les situations de soins et en viennent à les comprendre. Par extension, il est raisonnable de penser que le modèle recoupe certaines questions épistémologiques pertinentes pour le développement de la discipline infirmière en passant par une conception de la pratique qui se veut avant tout réflexive.

\section{Conclusion}

Cette étude a permis de traduire en français le modèle du jugement clinique infirmier de Tanner (2006) à l'aide d'une démarche structurée et par l'implication importante d'expertes en formation infirmière. La version française proposée dans cet article se veut un outil pour contribuer au développement de la science de la formation infirmière et à la bonification des pratiques de formation en soins infirmiers. 


\section{Références}

Ammon, U. (2011). The dominance of English as a language of science: Effects on other languages and language communities. Mouton de Gruyter. https://doi.org/10.1515/9783110869484

Banning, M. (2008). A review of clinical decision making: Models and current research. Journal of Clinical Nursing , 17, 187-195. https://doi.org/10.1111/j.1365-2702.2006.01791.x

Beauchamp, J. Lalonde, M., Fournier, V., Mehiz, S., Pedrotti, M., Michel, I., Godbout, P., et Simoneau, I. (2021). Traduction et adaptation française du Lasater Clinical Judgement: Une étude multicentrique. Quality Advancement in Nursing Education - Avancées en formation infirmière, 7(2).

Benner, P. (1984). From novice to expert: Excellence and power in clinical practice. AddisonWesley.

Benner, P. E., Tanner, C. A., et Chesla, C. A. (2009). Expertise in nursing practice: Caring, clinical judgment, and ethics ( $2^{\mathrm{e}}$ éd.). Springer.

Boyer, L., Tardif, J., et Lefebvre, H. (2015). From a medical problem to a health experience: How nursing students think in clinical situations. Journal of Nursing Education, 54(11), 625-632. https://doi.org/10.3928/01484834-20151016-03

Cappelletti, A., Engel, J. K., et Prentice, D. (2014, août). Systematic review of clinical judgment and reasoning in nursing. Journal of Nursing Education, 53(8), 453-458. https://doi.org/10.3928/01484834-20140724-01

Deschênes, M.-F., et Goudreau, J. (2017). Addressing the development of both knowledge and clinical reasoning in nursing through the perspective of script concordance: An integrative literature review. Journal of Nursing Education and Practice, 7(12), 29-38. https://doi.org/https://doi.org/10.5430/jnep.v7n12p28

Dewey, J. (1933). How we think: A restatement of the relation of reflective thinking to the education process. Heath.

Diekelman, N., et Ironside, P. M. (2002). Developing a science of nursing education: Innovation with research. Journal of Nursing Education, 41, 379-380. https://doi.org/10.3928/01484834-20020901-03

Dreyfus, S. E., et Dreyfus, H. L. (1980). A five-stage model of the mental activities involved in directed skills acquisition. https://apps.dtic.mil/dtic/tr/fulltext/u2/a084551.pdf

Dubé, V., et Ducharme, F. (2015). Nursing reflective practice: An empirical literature review. Journal of Nursing Education and Practice, 5(7), 91-99. https://doi.org/10.5430/jnep.v5n7p91

Evans, J. S. (2008). Dual-processing accounts of reasoning, judgment, and social cognition. Annual Review of Psychology, 59, 255-278. https://doi.org/10.1146/annurev.psych.59.103006.093629

Goudreau, J., Boyer, L., et Létourneau, D. (2014). Clinical nursing reasoning in nursing practice: A cognitive learning model based on a think aloud methodology. Quality Advancement in Nursing Education - Avancées en formation infirmière, 1(1). https://doi.org/10.17483/2368-6669.1009 
Goudreau, J., Pepin, J., Larue, C., Dubois, S., Descoteaux, R., Lavoie, P., et Dumont, K. (2015). A competency-based approach to nurses' continuing education for clinical reasoning and leadership through reflective practice in a care situation. Nurse Education in Practice, 15(6), 572-578. https://doi.org/10.1016/j.nepr.2015.10.013

Goulet, M.-H., Larue, C., et Alderson, M. (2016). Reflective practice: A comparative dimensional analysis of the concept in nursing and education studies. Nursing Forum, 51(2), 139-150. https://doi.org/10.1111/nuf.12129

Gresley, R. S. (2009). Building a science of nursing education. Dans C. Shultz (dir.), Building a science of nursing education: Foundation for evidence-based teaching-learning (p. 1-13). National League for Nursing.

Hammond, K. R. (1996). Human judgment and social policy: Irreducible uncertainty, inevitable error, unavoidable injustice. Oxford University Press.

Hammond, K. R., Hamm, R. M., Grassia, J., et Pearson, T. (1987). Direct comparison of the efficacy of intuitive and analytical cognition in expert judgment. IEEE: Transations on Systems, Man, and Cybernetics, 17(5), 753-770. https://doi.org/10.1109/TSMC.1987.6499282

Howard, R. A. (1966). Decision analysis: Applied decision theory. Stanford Research Institute.

Huang, H. M., Huang, C. Y., Lee-Hsieh, J., et Cheng, S. F. (2018). Establishing the competences of clinical reasoning for nursing students in Taiwan: From the nurse educators' perspectives. Nurse Education Today, 66, 110-116. https://doi.org/10.1016/j.nedt.2018.04.007

Huang, Y. C., Chen, H. H., Yeh, M. L., et Chung, Y. C. (2012, juin). Case studies combined with or without concept maps improve critical thinking in hospital-based nurses: A randomized-controlled trial. International Journal of Nursing Studies, 49(6), 747-754. https://doi.org/10.1016/j.ijnurstu.2012.01.008

Kahneman, D. (2011). Thinking, fast and slow (1 $1^{\text {re }}$ éd.). Farrar, Straus and Giroux. http://catalogue.bnf.fr/ark:/12148/cb42708522k

Lasater, K. (2007a). Clinical judgment development: Using simulation to create an assessment rubric. Journal of Nursing Education, 46(11), 496-503. https://doi.org/https://doi.org/10.3928/01484834-20071101-04

Lasater, K. (2007b). High-fidelity simulation and the development of clinical judgment: Students' experiences. Journal of Nursing Education, 46(6), 269-276. https://doi.org/10.3928/01484834-20070601-06

Lasater, K., Holloway, K., Lapkin, S., Kelly, M., McGrath, B., Nielsen, A., Stoyles, S., Dieckmann, N. F., et Campbell, M. (2019). Do prelicensure nursing students' backgrounds impact what they notice and interpret about patients? Nurse Education Today, 78, 37-43. https://doi.org/10.1016/j.nedt.2019.03.013

Lavoie, P., Boyer, L., Pepin, J. I., Goudreau, J., et Fima, O. (2017). Accompagner les infirmières et les étudiantes dans la réflexion sur des situations de soins : Un modèle pour les formateurs en soins infirmiers. Quality Advancement in Nursing Education - Avancées en formation infirmière, 3(1). https://doi.org/10.17483/2368-6669.1100 
Lee, J., Lee, Y. J., Bae, J., et Seo, M. (2016). Registered nurses' clinical reasoning skills and reasoning process: A think-aloud study. Nurse Education Today, 46, 75-80. https://doi.org/10.1016/j.nedt.2016.08.017

Levett-Jones, T., Hoffman, K., Dempsey, J., Jeong, S. Y. S., Noble, D., Norton, C. A., Roche, J., et Hickey, N. (2010). The 'five rights' of clinical reasoning: An educational model to enhance nursing students' ability to identify and manage clinically 'at risk' patients. Nurse Education Today, 30(6), 515-520. https://doi.org/10.1016/j.nedt.2009.10.020

McCarthy, M. (2003). Situated clinical reasoning: distinguishing acute confusion from dementia in hospitalized older adults. Reseach in Nursing \& Health, 26(2), 90-101. https://doi.org/10.1002/nur.10079

Newell, A., et Simon, H. A. (1972). Human problem solving. Prentice-Hall.

Nielsen, A., Stragnell, S., et Jester, P. (2007). Guide for reflection using the clinical judgment model. Journal of Nursing Education, 46(11), 513-516. https://doi.org/10.3928/01484834-20071101-06

O’Neill, E. S., Dluhy, N. M., et Chin, E. (2005). Modelling novice clinical reasoning for a computerized decision support system. Journal of Advanced Nursing, 49(1), 68-77. https://doi.org/10.1111/j.1365-2648.2004.03265.X

Oermann, M. H. (2007). Approaches to gathering evidence for educational practices in nursing. Journal of Continuing Education in Nursing, 38(6), 250-255. https://doi.org/10.3928/00220124-20071101-05

Pepin, J., Ducharme, F., et Kérouac, S. (2017). La pensée infirmière (4éd.). Chenelière éducation.

Pesut, D. J., et Herman, J. (1998). OPT: Transformation of nursing process for contemporary practice. Nursing Outlook, 46(1), 29-36. https://doi.org/10.1016/s0029-6554(98)90022-7

Simmons, B. (2010). Clinical reasoning: Concept analysis. Journal of Advanced Nursing, 66(5), 1151-1158. https://doi.org/10.1111/j.1365-2648.2010.05262.x

Sousa, V. D., et Rojjanasrirat, W. (2011, avril). Translation, adaptation and validation of instruments or scales for use in cross-cultural health care research: A clear and userfriendly guideline. Journal of Evaluation in Clinical Practice, 17(2), 268-274. https://doi.org/10.1111/j.1365-2753.2010.01434.x

Tanner, C. A. (2006). Thinking like a nurse: A research-based model of clinical judgment in nursing. Journal of Nursing Education, 45, 204-211. https://doi.org/https://doi.org/10.3928/01484834-20060601-04

Thompson, C., Aitken, L., Doran, D., et Dowding, D. (2013, décembre). An agenda for clinical decision making and judgement in nursing research and education. International Journal of Nursing Studies, 50, 1720-1726. https://doi.org/10.1016/j.ijnurstu.2013.05.003

Von Neumann, J., et Morgenstern, O. (1947). Theory of games and economic behavior (2 éd.). Princeton University Press. 